\title{
Recent progress in development and application of sub-THz gyrotrons in University of Fukui
}

\author{
$\underline{\text { Y. Tatematsu }}$ \\ Research Center for Development of Far-infrared Region, University of Fukui, Fukui, Japan, tatema@fir.u-fukui.ac.jp
}

\section{Introduction}

Till 2005, Research Center for Development of Far-Infrared Region, University of Fukui (herein referred to as FIR-UF) had developed gyrotrons to aim at increasing their frequency. For this purpose, the Gyrotron FU series were developed[1-3]. Fundamental, second and third harmonic oscillations were observed, and the breakthrough of $1 \mathrm{THz}$ oscillation in gyrotron was achieved for the first time in the world[4].

Since 2006, gyrotrons have been developed in FIR-UF for the purpose of applications such as DNPNMR spectroscopy[5,6], sintering and direct measurement of energy level of positronium hyperfine structure[7]. Gyrotrons developed for this purpose are Gyrotron FU CW series[8-13], Gyrotron FU CW G series[14-19] and pulsed gyrotrons[20-28].

\section{Development of Gyrotron}

Gyrotron FU CW I was developed in the collaboration between IAP, RAS and FIR-UF[10]. It was fabricated by Gycom. It was used for material processing and sintering. Some gyrotrons applying for DNP-NMR spectroscopies were developed, such as Gyrotrons FU CW II, IV, VI and VII[9,11-13]. For this application, the frequency tuning is necessary to adjust the sensitivity to maximum. With Gyrotron FU CW IV, a continuous frequency variation width of 6 $\mathrm{GHz}$ was observed[11]. The oscillation mode was fundamental $\mathrm{TE}_{12}$ mode. The mechanism of continuous frequency variation was explained as the interaction with gyro-backward waves.

Recently Gyrotron FU CW XA was developed. It is installed on an $8 \mathrm{~T}$ superconducting magnet. It is a linear type gyrotron without a built-in mode converter. The cavity is a normal hollow one with up and down tapers at the both ends of the straight section. The radius and length of the straight section of the cavity are $2.997 \mathrm{~mm}$ and $20 \mathrm{~mm}$, respectively. Although this cavity was not specially designed for the purpose of frequency tuneability, frequency variations more than $1 \mathrm{GHz}$ were measured with four modes, $\mathrm{TE}_{12}, \mathrm{TE}_{13}$, $\mathrm{TE}_{33}$ and $\mathrm{TE}_{14}$, in this gyrotron. The frequency bands are 90, 140, 180, and $190 \mathrm{GHz}$, respectively[29].

For applications, conversion to Gaussian beam is necessary. About Gaussian beam output gyrotrons in FIR-UF, the Gyrotron FU CW I is the first, which was fabricated in Russia[10]. Gaussian beam output gyrotrons developed in FIR-UF are called as Gyrotron FU CW G series[14-19]. Gyrotron FU CW GI oscillates in $203 \mathrm{GHz}$ frequency[14] and was used for direct measurement of the energy level of positoronium hyperfine structure[7]. Gyrotrons FU CW GII and GIII oscillate in second harmonic[15-17]. Gyrotron FU
CW GIV showed frequency variations in two frequency ranges. The frequency varies with the widths of $5 \mathrm{GHz}$ in $190 \mathrm{GHz}$ band for the fundamental mode and of $3 \mathrm{GHz}$ in $390 \mathrm{GHz}$ band for the second harmonic mode[30].

Gyrotron FU CW GV is a multi-frequency gyrotron[18]. It is of sealed-off type and was constructed by New Japan Radio Company. It was installed on a $10 \mathrm{~T}$ magnet. The mode converter consists of a conventional Vlasov launcher and four mirrors. Although it was designed for the $\mathrm{TE}_{10,6}$ mode, Gaussian beams were radiated in oscillations of ten modes. Their oscillation frequencies are from $162 \mathrm{GHz}$ to $265 \mathrm{GHz}$ about $10 \mathrm{GHz}$ step. The transmittance of a window disk depends on the wave frequency. Some of them have low transmittance. To prevent the drop in transmittance due to the frequency-dependence, a distance variable double-disk window was adopted. With variation of the distance between the two disks, the transmittance of the electromagnetic wave periodically changed because of the wave interference. The transmittance can be almost one by setting with appropriate distances for every frequency.

In Gyrotrons FU CW VI and VIA, frequency variation around $460 \mathrm{GHz}$ was achieved in a second harmonic oscillation using a $10 \mathrm{~T}$ magnet[19].

We also developed pulsed high power gyrotron series[20-28]. They are developed in the purpose of a light source for the Collective Thomson Scattering (CTS) measurement in LHD, NIFS. First, we developed $400 \mathrm{GHz}$ frequency, 2nd harmonic oscillation gyrotrons using $8 \mathrm{~T}$ superconductive magnet. Howev$\mathrm{er}$, for the required target power of $100 \mathrm{~kW}$ injection into the plasma, 2nd harmonic oscillation did not reach the goal power. So, we changed to $300 \mathrm{GHz}$ gyrotron development using $12 \mathrm{~T}$ magnet in fundamental oscillation. With the beam voltage of $65 \mathrm{kV}$ and current of $15 \mathrm{~A}$, power of $320 \mathrm{~kW}$ and efficiency of 33 percent are achieved[28]. This gyrotron was moved to LHD of NIFS in July, 2018 and will be applied to CTS experiment.

\section{Applications of Gyrotron}

As examples of applications of sub-THz gyrotrons, there are sintering, DNP-NMR spectroscopy, positronium energy level measurement, CTS, pulsed ESR, millimeter wave discharge, light emission from crystals and study for safety data base of sub-THz radiation.

Gyrotron FU CW GI was applied for direct measurement of hyperfine structure of positronium[7]. This is a collaboration with Tokyo University. Positoronium is a bound state of a positron and an electron. Ground-state positronium has two spin eigenstates, 
ortho-state and para-state. The energy difference of the two states correspond to about $203.4 \mathrm{GHz}$. The measurements of the energy difference were done in the past, however, they were measurement used the Zeeman effect in a static magnetic field. It may be affected by the magnetic field, and the result has a slightly difference from the theoretically calculated values. So, we did directly measurement of the energy level using electromagnetic wave. The expected spectrum of the gamma ray annihilation probability is a function of frequency. So, for this measurement, a light source with frequency tunable between 201 to $206 \mathrm{GHz}$ and high power more than $100 \mathrm{~W}$ with Gaussian beam output is necessary. However, frequency of a gyrotron is not widely continuously changed to keep high power. Therefore, to change the frequency, we changed the cavity itself. We prepared a lot of cavities whose diameters were slightly different each other. Changing the cavities, the intensity of gamma-ray produced by pair annihilation was measured. The energy difference of the hyperfine structure of positronium was measured with the central energy of $203.39 \pm 0.11 \mathrm{GHz}$.

Another application of gyrotron is light emission from $\mathrm{ZnO}$ crystal[31]. This is a collaboration with Osaka University. Spectra of irradiation of a $\mathrm{ZnO}$ crystal by gyrotron exhibit a broad and asymmetric peak around $600 \mathrm{~nm}$ with a longer-wavelength tail. When the duty ratio of gyrotron irradiation increased, the peak emission intensity increases rapidly. Yellow light emitted from the $\mathrm{ZnO}$ crystal. The temperature was more than 1000 degree by the IR camera measurement. When heated up to $1250 \mathrm{~K}$ using an electric heater without gyrotron irradiation, the crystal does not exhibit any visible emission. Therefore, this result indicates that the yellow emission of the gyrotron irradiated crystal is not a simple blackbody radiation but is caused by a combination of thermal effects and the gyrotron's sub-THz electric field.

When sub-THz wave from a gyrotron is radiated to nano-scale cages of $\mathrm{Ca}_{12} \mathrm{Al}_{14} \mathrm{O}_{33}$ crystal, visible light was emitted from the crystal[32]. It was explained the mechanism of lightening that oxygen ions inside the cage rattle due to sub- $\mathrm{THz}$ wave and emit visible light. Conversion from $\mathrm{THz}$ wave to visible light is useful for $\mathrm{THz}$ range research to specify the wave position. This is a collaboration with Tokyo Institute of Technology and Hirosaki University.

Gyrotron is also applied for the study of safety data base of sub-THz radiation for eyes. This is a collaboration with Tokyo Metropolitan University and Kanazawa Medical University. They had the experiments at frequencies of $40,75,90 \mathrm{GHz}$ irradiation so far. In the present experiment, we use electromagnetic wave with the frequency of $162 \mathrm{GHz}$, one of oscillation of Gyrotron FU CW GV. Sub-THz wave is irradiated into rabbit eyes, and the threshold of power or power density to cause injury on the eyes is being investigated.

A pulsed high power gyrotron of $303 \mathrm{GHz}$ will be applied for CTS measurement in LHD, NIFS. The electromagnetic wave generated by a gyrotron is injected into plasma. From the observation of the scat- tering wave, we can obtain the information of ion temperture. Due to the magnetic field distribution of LHD, 300 or $400 \mathrm{GHz}$ is the best frequencies for CTS measurement in LHD.

\section{Summary}

In FIR-UF, Gyrotron FU CW series, Gyrotron FU CW G series and pulsed gyrotron series have been developed for the purpose of various applications for the current decade and they have been and will be applied for various fields.

\section{References}

1. Idehara, T. et al., Phys. Fluids B 5, 1377 (1993).

2. Hong, K. D. et al., J. Appl. Phys. 74, 5250 (1993).

3. Shimizu, Y. et al., Phys. Plasmas 2, 2110 (1995).

4. Idehara, T. et al., Int. J. Infrared. Milli. Waves. 27, 319 (2006).

5. Matsuki, Y. et al., J. Mag. Res. 225, 1 (2012).

6. Matsuki, Y. et al., J. Mag. Res. 264, 107 (2016).

7. Miyazaki, A. et al, Prog. Theo. Exp. Phys. 2015, 011C01 (2015)

8. La Agusu et al., Int. J. Infrared. Milli. Waves. 28, 315 (2007)

9. Idehara, T. et al., Int. J. Infrared. Milli. Waves. 28, 433 (2006).

10. Zapevalov, V. E. et al., Radiophys. Quant. Electron. 50, 420 (2007)

11. Chang T.-H. et al., j Appi. Phys. 105, 063304 (2009).

12. Idehara, T. et al., J. Infrared Milli. Terahertz Waves 31, 763 (2010).

13. Idehara, T. et al., J. Infrared Milli. Terahertz Waves 31, 775 (2010).

14. Tatematsu et al., J. Infrared Milli. Terahertz Waves 33, 292 (2012).

15. Tatematsu et al., J. Infrared Milli. Terahertz Waves 35, 169 (2014).

16. Tatematsu et al., J. Infrared Milli. Terahertz Waves 35, 517 (2014).

17. Tatematsu et al., Phys Plasmas 21, 083113 (2014).

18. Tatematsu et al., J. Infrared Milli. Terahertz Waves 36, 697 (2015).

19. Idehara, T. et al., J. Infrared Milli. Terahertz Waves 36, 819 (2015).

20. Notake, T. et al., Rev. Sci. Instrum. 79, 732 (2008).

21 Notake, T. et al., Plasma Fusion Res. 4, 011 (2009).

22. Notake, T. et al., Phys. Rev. Lett 103, 225002 (2009).

23. Saito, T. et al., Plasma Fusion Res. 7, 1206003 (2012).

24. Saito, T. et al., Phys. Plasmas 19, 063106 (2012).

25. Yamaguchi, Y. et al., Plasma Fus Res. 8, 1205165

(2013).

26. Yamaguchi, Y. et al., Nuclear Fusion 55, 13002 (2015).

27. Yamaguchi, Y. et al., J. Instrumentation 10, C10002, (2015).

28. Saito, T. et al., Plasma Fusion Res. 12, 1206013-11206013-2, 2017.

29. Tatematsu et al., 42nd IRMMW-THz, RA2.2, 2017.

30. Tatematsu et al., 41st IRMMW-THz, H5P.21.13, 2016.

31. Kato, K. et al., Appl. Phys. Lett. 111, 031108 (2017).

32. Toda, Y. et al., ACS Nano 11, 12358 (2017). 